\title{
Surface Reconstruction Induced by Transition Metal Doping of Rutile Titanium Dioxide (110)
}

\author{
Ralf Bechstein, ${ }^{\dagger}$ Mitsunori Kitta, ${ }^{\ddagger}, \S$ Jens Schütte, ${ }^{\dagger}$ Angelika Kühnle, ${ }^{*, \dagger}$ and Hiroshi Onishi ${ }^{\ddagger}$ \\ Fachbereich Physik, University of Osnabrück, Barbarastrasse 7, 49076 Osnabrück, Germany, and Department \\ of Chemistry, Kobe University, Rokko-dai, Nada-ku, Kobe 657-8501, Japan
}

Received: February 22, 2009; Revised Manuscript Received: April 7, 2009

\begin{abstract}
The influence of chromium and antimony codoping on the surface structure of rutile $\mathrm{TiO}_{2}(110)$ was studied at the atomic level using noncontact atomic force microscopy. Compared to pristine $\mathrm{TiO}_{2}(110)$, the codoped surface exhibits substantial surface facetting. A careful analysis of high-resolution images unraveled a surface reconstruction that closely resembles a $(1 \times 4)$ structure, indicating a firm integration of the dopant atoms into the titanium dioxide crystal. The reconstruction can be described by shearing the original unit cell by an angle of $7 \pm 1^{\circ}$. We provide a simple explanation of the observed reconstruction by comparing both the ionic as well as the covalent radii of chromium and antimony with those of titanium. On the basis of this simple picture, the observed reconstruction can be understood as a strategy to compensate for the significantly smaller covalent radii of the dopant atoms. The observed surface facetting is explained by reconstructioninduced interlayer stress. This facetting might be beneficial for photocatalytic activity. Our results thus shed light on the role of chromium and antimony dopants in the wide band gap photocatalyst titania.
\end{abstract}

\section{Introduction}

Titanium dioxide $\left(\mathrm{TiO}_{2}\right)$ represents an important photocatalyst that is used for degrading toxic compounds ${ }^{1}$ and has the potential for producing hydrogen fuel from water. ${ }^{2,3} \mathrm{TiO}_{2}$ is a wide band gap photocatalyst that is known to be sensitized to visible light by doping with foreign elements. ${ }^{4}$ Nonmetallic dopants have been examined and found to be active in visible-light induced reactions. ${ }^{5-10}$ Transition metal doping has been reported to result in even more intense visible-light absorption. ${ }^{11}$ While chromium doping has been demonstrated to result in visible-light absorption, photocatalytic activity has not been observed. ${ }^{12}$ The photocatalytic inactivity of chromium-doped $\mathrm{TiO}_{2}$ has been explained by the formation of oxygen vacancies and the presence of $\mathrm{Cr}^{6+}$ upon chromium doping, ${ }^{12,13}$ representing recombination sites for electron-hole pairs. This assumption has been confirmed by recent noncontact atomic force microscopy (NCAFM) measurements, ${ }^{14}$ revealing an increased density of oxygen vacancies on chromium-doped $\mathrm{TiO}_{2}(110)$. In contrast, when codoped with chromium and antimony both visible-light absorption and photocatalytic activity has been achieved. ${ }^{12,13}$ It has been argued that this might be due to the presence of $\mathrm{Cr}^{3+}$ and $\mathrm{Sb}^{5+}$ cations, resulting in charge neutrality with no need for oxygen vacancy or $\mathrm{Cr}^{6+}$ creation. ${ }^{12,13}$ However, experimental evidence for this assumption was lacking so far.

Here, we present an NC-AFM investigation of chromium and antimony codoped $\mathrm{TiO}_{2}(110)$, revealing insight into the influence of codoping on the surface structure at the atomic level. A doping-induced surface reconstruction is observed, indicating a firm integration of the dopants into the crystal lattice. Moreover, in contrast to what has been observed on $\mathrm{TiO}_{2}(110)$ doped with chromium solely, ${ }^{14}$ no increase in surface oxygen vacancy density is found on the codoped sample, supporting

* To whom correspondence should be addressed. E-mail: kuehnle@uos.de.

University of Osnabrück.

* Kobe University.

$\S$ Present Address: Department of Chemistry, School of Science, Osaka University, Mihogaoka, Ibaraki, Osaka, 567-0047 Japan. previous assumptions ${ }^{12,13}$ with clear-cut experimental evidence. We present a simple model based on the ionic and the covalent radii of the dopant atoms, which provides excellent agreement with the experimental findings.

\section{Experimental Section}

High-resolution NC-AFM was used operated in the frequency modulation mode. For this technique, a sharp nanoscopic tip mounted at the end of an oscillating cantilever was scanned in close proximity over the sample surface while keeping the oscillation amplitude constant. The forces acting between tip and surface atoms shifted the cantilever's eigenfrequency. This frequency shift was directly used as an imaging signal when scanning the cantilever at a fixed tip-surface distance. Alternatively, the frequency shift was kept constant to measure the height profile of equal frequency shift (referred to as topography). Measurements were performed at room temperature in an ultrahigh vacuum chamber. The experimental setup was the same as described previously. ${ }^{14,15} \mathrm{The}^{\mathrm{TiO}_{2}}$ (110) samples were crystals of highest quality available (MTI, Richmond). The crystals were codoped with chromium and antimony by calcining them together with $\mathrm{Cr}_{2} \mathrm{O}_{3}$ and $\mathrm{Sb}_{2} \mathrm{O}_{3}$ powder in a crucible at $1420 \mathrm{~K}$ for $10 \mathrm{~h}$ in air as described for titania powder. ${ }^{12,13}$ The dopant ratio was intended to be unity in the doped single crystal. ${ }^{12}$ The concentrations of chromium and antimony in the doped titanium dioxide single crystal cannot be expected to be homogeneous and was therefore difficult to quantify. However, in the upper few micrometers the chromium and antimony concentrations were expected to be in the order of 2 to 3 at. \% as observed in similarly doped titania powder. ${ }^{12}$ This order of magnitude was confirmed by an X-ray photoelectron spectroscopy (XPS) measurement of the freshly calcined wafer. The dopant ratio was estimated from the XPS data to be about [Cr]/ $[\mathrm{Sb}]=3 / 5$. On the basis of these numbers, the total dopant concentration $[\mathrm{Cr}]+[\mathrm{Sb}]$ can be estimated to be in the range of $3-8$ at. $\%$. The rather large error reflects the above-described uncertainties. After insertion into the UHV chamber, the surface 

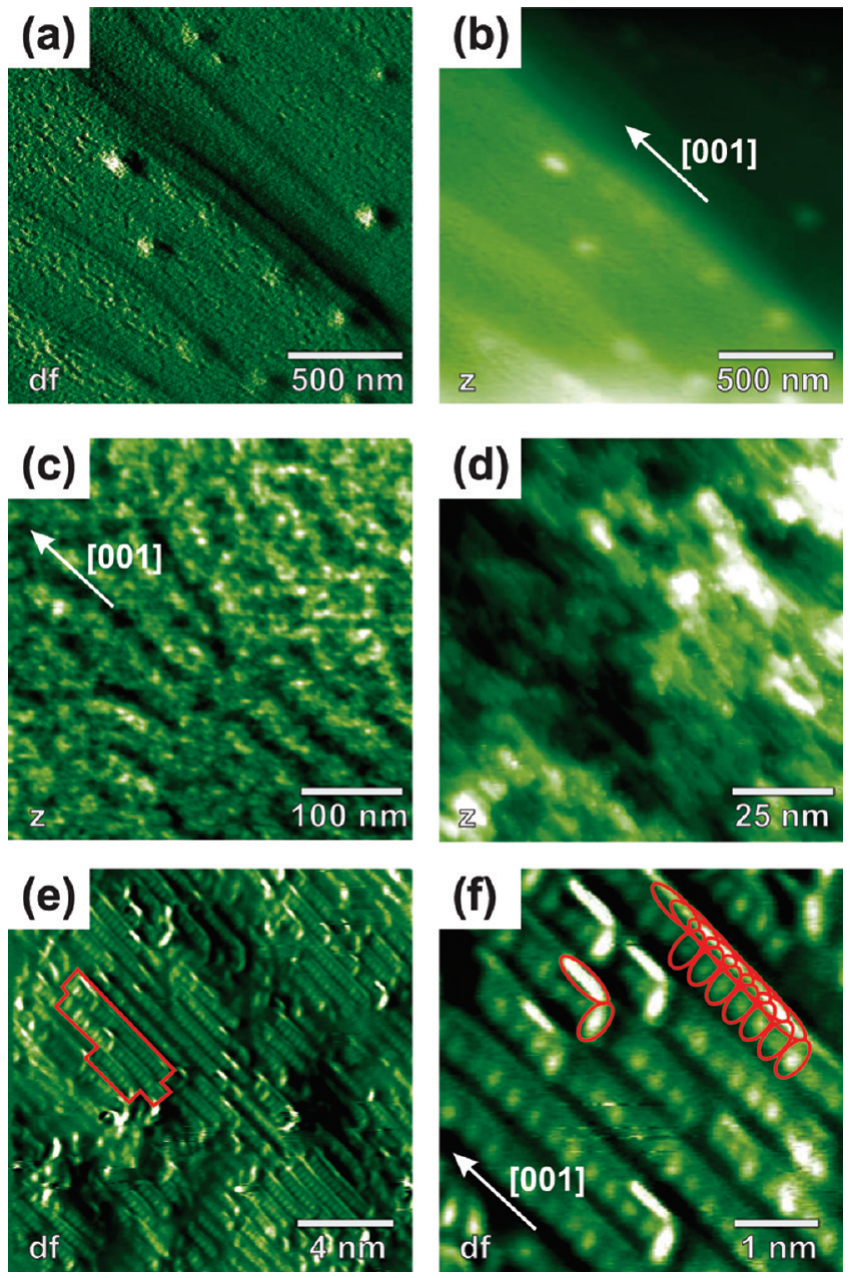

Figure 1. NC-AFM topography $(\mathrm{z})$ and frequency shift (df) images of a $\mathrm{TiO}_{2}(110)$ sample codoped with chromium and antimony. $(\mathrm{a}, \mathrm{b})$ An overview revealing high steps aligned in [001] direction. The regions in between are rough areas with a lot of troughs (c). (d) Hundreds of small, atomically flat facets can be identified. One of them is indicated in (e) by a red frame. True atomic resolution can be obtained on each facet, revealing bright atomic rows and protruding defects (e). In this particular case, an L-shaped tip results in additional bright lines next to each atomic row as indicated in (f) by red ellipses.

was cleaned by cycles of $15 \mathrm{~min} \mathrm{Ar}^{+}$ion sputtering at $1 \mathrm{keV}$ and subsequent annealing. The preparation parameter were chosen in accordance with our experience on pristine $\mathrm{TiO}_{2}(110) .{ }^{15} \mathrm{In}$ order to minimize long-range electrostatic interactions, a bias voltage of about $(-1.0 \pm 0.1) \mathrm{V}$ was applied to the tip.

\section{Results and Discussion}

In this paper, we study the influence of transition metal doping on the surface structure of rutile $\mathrm{TiO}_{2}(110)$. An overview of NC-AFM images of decreasing frame size obtained on a $\mathrm{TiO}_{2}(110)$ surface codoped with chromium and antimony is shown in Figure 1. At large frame size (Figure 1a,b), steplike structures with a typical height of $1-10 \mathrm{~nm}$ are observed. The regions in between these steps exhibit a rough surface structure with a lot of troughs as can be seen in Figure 1c. The depth of these troughs is typically three to six times as large as a single monatomic step height on $\mathrm{TiO}_{2}(110)$. Extended, atomically flat terraces were never observed. Figure 1d,e reveals that the surface consists of numerous atomically flat facets. Each facet covers an area of typically $40 \mathrm{~nm}^{2}$. Thus, a typical facet consists of only a few hundred unit cells. This is in contrast to the equally stepped surface usually observed on similarly prepared pristine $\mathrm{TiO}_{2}(110)$, where terrace areas are larger by at least a factor of thousand.

To exclude that the surface roughness is just a result of inadequate preparation parameters, we systematically varied annealing temperature from 500 to $1100 \mathrm{~K}$ and annealing duration from $15 \mathrm{~min}$ up to $16 \mathrm{~h}$. Figure 2 shows three representative sets of images corresponding to three selected annealing recipes. The observed surface roughness and faceting is found to be virtually independent of the annealing parameters. Neither high temperature nor drastically increased annealing duration led to extended, atomically flat terraces.

At the atomic scale (Figure 1e,f), rows of bright atoms divided by thin, bright lines become visible. These thin, bright lines can be explained by a peculiar tip shape that images all surface species in a characteristic L-shape as indicated in Figure 1f. True atomic resolution has been obtained on nearly all facets simultaneously, although several monatomic steps typically exist in a single image. Single defects appear as protrusions on the atomic rows. The observed protrusions can be attributed to hydroxyl species as usually observed on the pristine surface, as the presented images were taken at least 30 min after sample preparation. ${ }^{16-20}$ Thus, we attribute the bright atoms to bridging oxygen atoms. The density of hydroxyls was estimated to be $(0.5 \pm 0.3) \%$ monolayer $(\% \mathrm{ML} ; 1 \mathrm{ML}$ corresponds to one per unit cell). From a previous defect density study on pristine titania we expect the density of hydroxyls to be $(0.9 \pm 0.6) \%$ $\mathrm{ML}^{15}$ on a similarly treated pristine sample, which is in good agreement with our present finding. Hence, dopant-induced bulk reduction as reported for chromium-doped $\mathrm{TiO}_{2}(110)^{14}$ is not observed on the codoped surface. At a first glance, the atomic structure of the doped surface resembles the structure of pristine $\mathrm{TiO}_{2}(110)$. Figure 3 shows subsequently recorded frequency shift images with true atomic resolution on several facets. The images were carefully corrected for constant thermal drift using identical drift velocity values for all images. The values of the drift velocity were obtained from a series of nine consecutively recorded images in total.

Determining the surface unit cell in the images in Figure 3, we found that the surface unit cell is not rectangular as for pristine $\mathrm{TiO}_{2}(110)$, but shows a surface reconstruction, indicating a firm integration of the dopant atoms into the titanium dioxide crystal structure. The surface unit cell is sheared by an angle of $7 \pm 1^{\circ}$ with respect to the surface unit cell of rutile. This shearing results in an appearance that closely resembles a $(1 \times$ 4) reconstruction. The findings are independent of the scan direction. Thus, we can definitely exclude scan artifacts or drift to be the origin of the shearing. In the following, we present an explanation for the observed experimental findings.

In codoped titania, chromium and antimony are expected to exist predominantly in the oxidation states $\mathrm{Cr}^{3+}$ and $\mathrm{Sb}^{5+}$, respectively. ${ }^{13}$ They substitute $\mathrm{Ti}^{4+}$ in the lattice. As long as the dopant ratio is close to unity, charging of the crystal does not take place. The ionic radii of the three cations are given in Table 1 . The values are nearly identical, pointing to a very similar bond length between the three metal species and oxygen. In a purely ionic picture, codoping of rutile titania with an equal amount of antimony and chromium would, therefore, not induce stress into the bulk. Hence, no reconstruction would be expected from this point of view.

However, titania is not a purely ionic crystal and the interaction is not purely electrostatic. Even though it is clear that titanium dioxide is rather ionic than covalent, minor effects like differences in covalence have to be taken into account to 


\section{$400 \times 400 \mathrm{~nm}^{2}$}

$40 \times 40 \mathrm{~nm}^{2}$
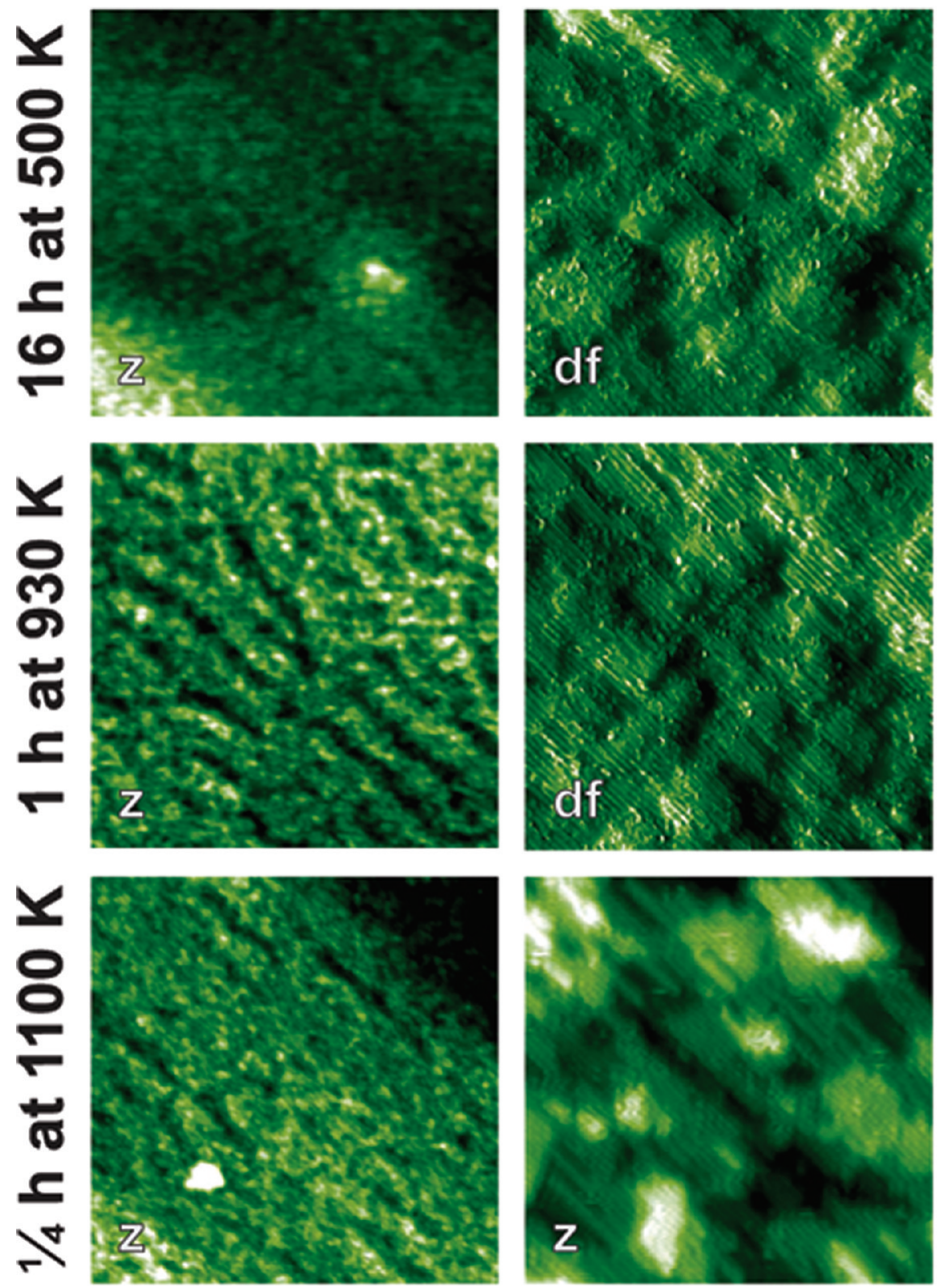

\section{$16 \times 16 \mathrm{~nm}^{2}$}
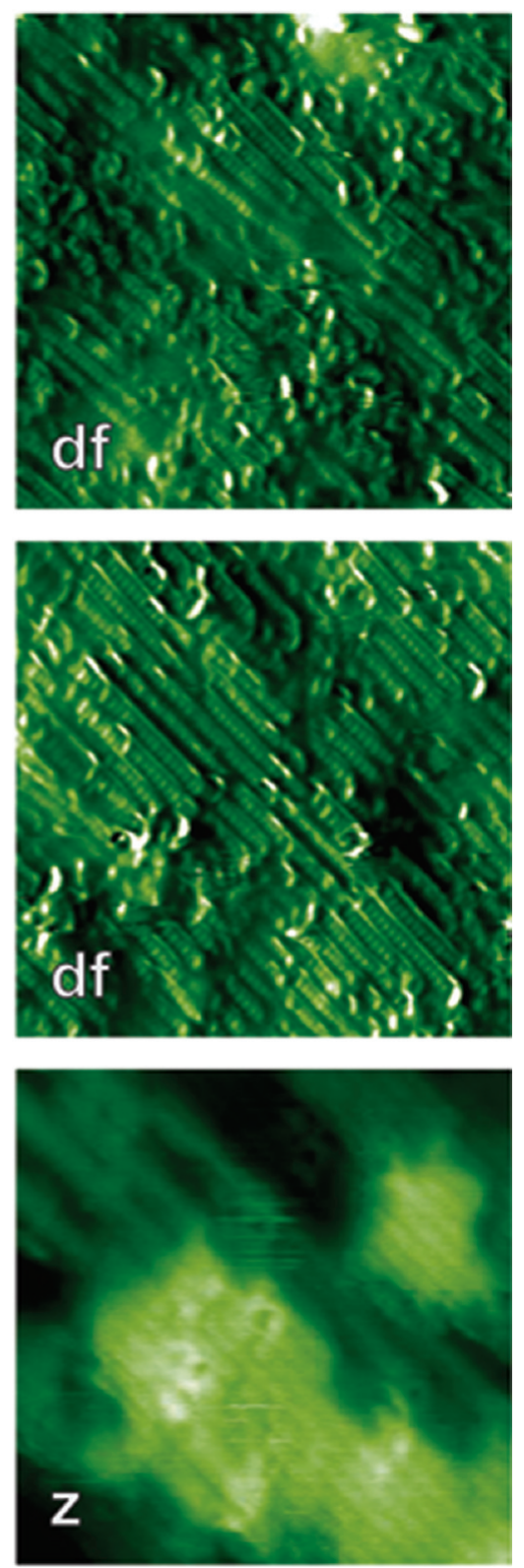

Figure 2. NC-AFM topography (z) and detuning (df) images of a codoped $\mathrm{TiO}_{2}(110)$ sample representing three annealing recipes. Images of equal size reveal qualitatively similar appearance. Hence, changing annealing temperature and duration does not change the observed surface roughness and faceting.

understand the observed surface reconstruction. The covalence of the metal-oxygen bond rather strongly depends on the metal species as can be seen from the electronegativity values also given in Table 1. The electronegativity difference between oxygen (3.44) and the dopant species is much smaller especially in the case of antimony than between titanium and oxygen. This clearly indicates a more covalent interaction between the dopants and oxygen than between titanium and oxygen in the pristine sample. Consequently, the covalent radii of chromium and antimony should be considered, which differ significantly from that of titanium. This discrepancy in covalence may explain the experimentally observed surface reconstruction. The correlation between doping and surface reconstruction will be discussed in the following model.

The surface unit cell of pristine $\mathrm{TiO}_{2}(110)$ contains one titanium atom with a covalent radius of $160 \mathrm{pm}$ and two oxygen atoms with covalent radii of $66 \mathrm{pm}^{22}$ The resulting covalent bond length for $\mathrm{Ti}-\mathrm{O}$ is $226 \mathrm{pm}$. (This simple model does not take into account that titanium is 5-fold or 6-fold coordinated on the titania surface. Thus, the exact bond lengths of 195 and $198 \mathrm{pm}^{16}$ are not precisely reproduced by a simple addition of the covalent radii. However, the following argumentation is independent of this discrepancy.) The $\mathrm{Ti}-\mathrm{O}$ bond length is a measure for the space a $\mathrm{Ti}-\mathrm{O}$ unit requires. Thus, this value is proportional to the square root of the unit cell area $A_{0}$ of rutile $\mathrm{TiO}_{2}(110)$

$$
\sqrt{A_{0}} \propto 160 \mathrm{pm}+66 \mathrm{pm}=226 \mathrm{pm}
$$

In the codoped sample, antimony and chromium atoms of smaller covalent radii substitute some of the titanium atoms. 

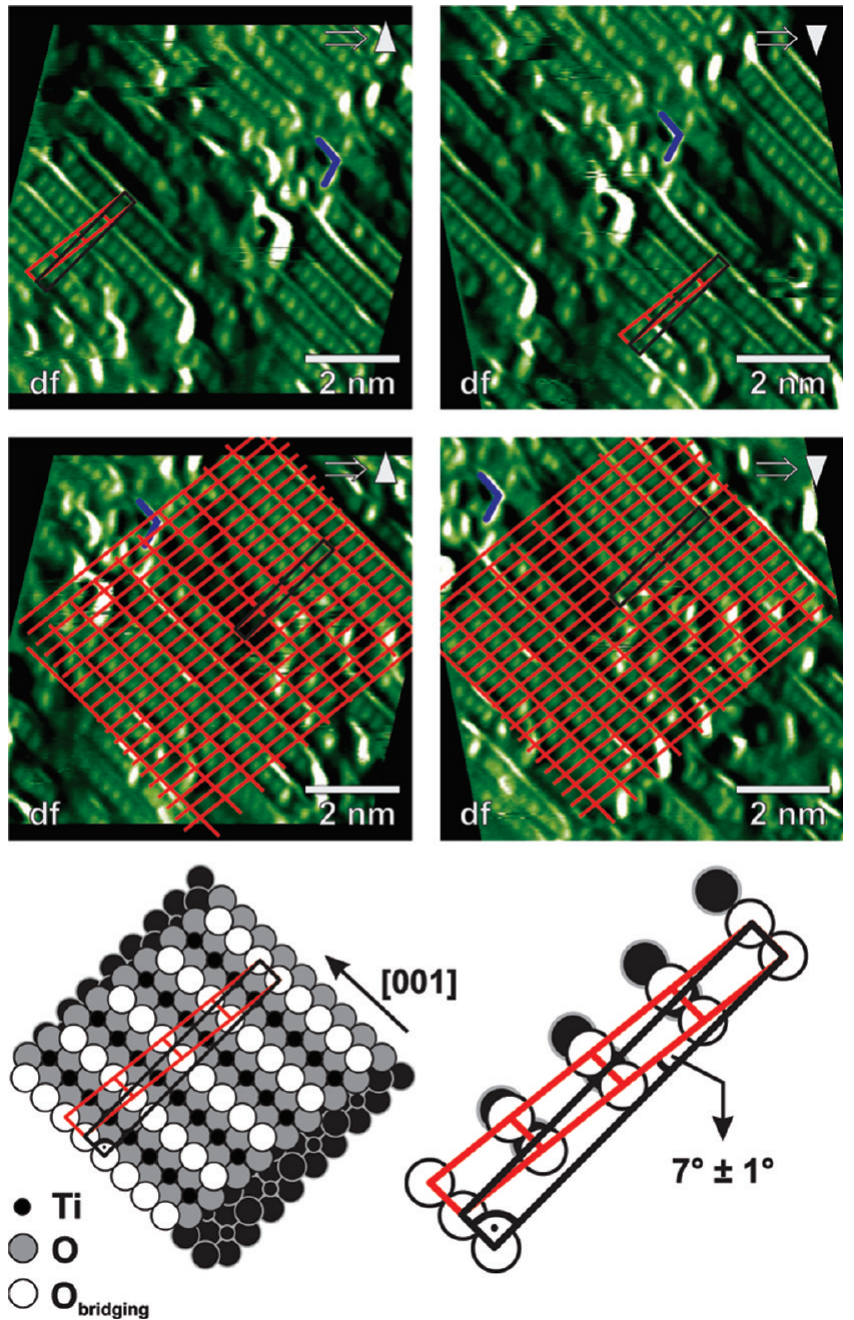

Figure 3. Subsequently taken NC-AFM frequency shift images carefully corrected for constant thermal drift using identical drift velocity values for all images (leading to nonrectangular images). The observed surface unit cell (indicated by red grid) is sheared by $7 \pm 1^{\circ}$ with respect to the surface unit cell of rutile $\mathrm{TiO}_{2}(110)$ (indicated by black rectangle). This appears very close to a $(1 \times 4)$ reconstruction.

TABLE 1: Electronegativity ${ }^{21}$ (Pauling Scale) and Covalent Radii $^{22}$ of Titanium and the Two Dopants as well as the Most Probable Oxidation State and the Ionic Radii of These Metal Ions $^{23}$

\begin{tabular}{ccccc}
\hline element & electronegativity & $\begin{array}{c}\text { covalent } \\
\text { radius }\end{array}$ & $\begin{array}{c}\text { oxidation } \\
\text { state }\end{array}$ & $\begin{array}{c}\text { ionic } \\
\text { radius }\end{array}$ \\
\hline $\mathrm{Ti}$ & 1.54 & $160 \mathrm{pm}$ & $\mathrm{Ti}^{4+}$ & $60.5 \mathrm{pm}$ \\
$\mathrm{Cr}$ & 1.66 & $139 \mathrm{pm}$ & $\mathrm{Cr}^{3+}$ & $61.5 \mathrm{pm}$ \\
$\mathrm{Sb}$ & 2.05 & $139 \mathrm{pm}$ & $\mathrm{Sb}^{5+}$ & $60.0 \mathrm{pm}$
\end{tabular}

Consequently, the average covalent bond length is reduced in codoped titania according to

$$
\begin{array}{r}
(1-[\mathrm{Cr}]-[\mathrm{Sb}]) 160 \mathrm{pm}+([\mathrm{Cr}]+[\mathrm{Sb}]) 139 \mathrm{pm}+ \\
66 \mathrm{pm}=226 \mathrm{pm}-([\mathrm{Cr}]+[\mathrm{Sb}]) 21 \mathrm{pm}
\end{array}
$$

where $[\mathrm{Cr}]$ and $[\mathrm{Sb}]$ are the atom concentrations of chromium and antimony, respectively. Eventually, the area of the surface unit cell is expected to be smaller than that of the pristine surface. Shearing of the unit cell reduces the area of the unit cell by the cosine of the shearing angle. In analogy to the case of pristine titania, we assume the square root of the surface unit cell area $A$ of the codoped

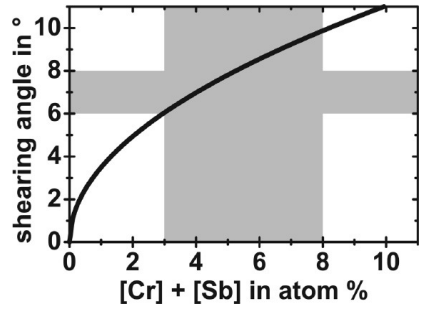

Figure 4. Shearing angle of surface unit cell of codoped $\mathrm{TiO}_{2}(110)$ in dependence of the total dopant concentration $[\mathrm{Cr}]+[\mathrm{Sb}]$. The calculation (solid line) only takes into account the dopant-induced reduced covalent bond length. The range of expected total dopant concentration (3-8 at. \%) as well as the experimentally observed shearing angle $\left(7 \pm 1^{\circ}\right)$ are indicated by gray boxes.

surface to be proportional to the average covalent bond length. With the values from Table 1 we obtain

$$
\sqrt{A}=\sqrt{A_{0} \cos \alpha} \propto 226 \mathrm{pm}-([\mathrm{Cr}]+[\mathrm{Sb}]) 21 \mathrm{pm}
$$

where $\alpha$ is the shearing angle. Substituting $A_{0}$ by the above formula, we derive

$$
\cos \alpha=\left(1-\frac{21}{226}([\mathrm{Cr}]+[\mathrm{Sb}])\right)^{2}
$$

for the cosine of the shearing angle in dependence on the dopant concentrations. The result is plotted in Figure 4.

Our simple model predicts a shearing angle of about $8 \pm 2^{\circ}$ to balance a total dopant concentration of $3-8$ at. $\%$ as present in the experiment. Within the error, this is exactly the shearing angle observed in the NC-AFM images.

The strikingly good agreement between our observations and the angle derived from this simple model supports the assumption of a firm integration of the dopant atoms into the titanium dioxide crystal structure. On the basis of our simple picture, the observed reconstruction can be understood as a strategy to compensate for the significantly smaller covalent radii of the dopant atoms. Note, however, that although the shearing angle of about $7^{\circ}$ can be measured quite accurately, the resulting relative length deviation of as small as $\left(1-\cos 7^{\circ}\right)=0.007$ is far beyond the accuracy of our lateral dimension measurement. Thus, it is not possible based on these measurements to conclude unambiguously that the unit cell is indeed compressed. However, to give a possible explanation of the observed reconstruction, we assume that the unit cell area becomes smaller by shearing. The shearing angle and therefore the reconstruction are experimental facts without any doubt; strongly supporting the presented model.

The small size of facets compared to pristine titania might result from stress between the reconstructed surface layer and underlying layers. Thus, the roughness of the surface is intrinsically tied to the codoping and cannot be overcome by any preparation recipe.

\section{Conclusion}

In conclusion, chromium and antimony codoping of rutile $\mathrm{TiO}_{2}(110)$ results in a surface reconstruction, indicating a firm integration of the dopant atoms, in sharp contrast to what is observed for chromium doping solely. ${ }^{14}$ An increased oxygen vacancy density as known for chromium doping is not observed, explaining why codoping with antimony recovers photocatalytic activity. The observed reconstruction can be understood as a strategy to compensate for the significantly smaller covalent radii 
of the dopant atoms. This reconstruction might explain the observed surface facetting by reconstruction-induced interlayer stress. As the surface morphology is known to be decisive for photocatalytic activity, the reconstruction might be beneficial for catalysis applications.

Acknowledgment. Financial support from the Deutsche Forschungsgemeinschaft (DFG) through an Emmy Noether grant is gratefully acknowledged. The present work was partly supported by a Grant-in-Aid for Scientific Research on Priority Areas [477] "Molecular Science for Supra Functional Systems" from the Ministry of Education, Culture, Sports, Science, and Technology, Japan.

\section{References and Notes}

(1) Fujishima, A.; Rao, T. N.; Tryk, D. A. J. Photochem. Photobiol. C 2000, 1, 1-21.

(2) Fujishima, A.; Honda, K. Nature 1972, 238, 37-38.

(3) Linsebigler, A. L.; Lu, G.; Yates, J. T. Chem. Rev. 1995, 95, 735758.

(4) Chen, X.; Mao, S. S. Chem. Rev. 2007, 107, 2891-2959. 773

(5) Irie, H.; Watanabe, Y.; Hashimoto, K. Chem. Lett. 2003, 32, 772-

(6) Asahi, R.; Morikawa, T.; Ohwaki, T.; Aoki, K.; Taga, Y. Science 2001, 293, 269-271.

(7) Batzill, M.; Morales, E. H.; Diebold, U. Phys. Rev. Lett. 2006, 96 , 026103

(8) Czoska, A. M.; Livraghi, S.; Chiesa, M.; Giamello, E.; Agnoli, S.; Granozzi, G.; Finazzi, E.; Di Valentin, C.; Pacchioni, G. J. Phys. Chem. C 2008, 112, 8951-8956.
(9) Lin, L.; Lin, W.; Zhu, Y.; Zhao, B.; Xie, Y. Chem. Lett. 2005, 34 , 284-285.

(10) Ohno, T.; Mitsui, T.; Matsumura, M. Chem. Lett. 2003, 32, 364365.

(11) Di Paola, A.; Marcì, G.; Palmisano, L.; Schiavello, M.; Uosaki, K.; Ikeda, S.; Ohtani, B. J. Phys. Chem. B 2002, 106, 637-645.

(12) Kato, H.; Kudo, A. J. Phys. Chem. B 2002, 106, 5029-5034.

(13) Ikeda, T.; Nomoto, T.; Eda, K.; Mizutani, Y.; Kato, H.; Kudo, A.; Onishi, H. J. Phys. Chem. C 2008, 112, 1167-1173.

(14) Bechstein, R.; Kitta, M.; Schütte, J.; Kühnle, A.; Onishi, H. J. Phys. Chem. C 2009, 113, 3277-3280.

(15) Schütte, J.; Bechstein, R.; Rahe, P.; Langhals, H.; Rohlfing, M.; Kühnle, A. Phys. Rev. B 2009, 79, 045428.

(16) Diebold, U. Surf. Sci. Rep. 2003, 48, 53-229.

(17) Wendt, S.; Schaub, R.; Matthiesen, J.; Vestergaard, E. K.; Wahlstroem, E.; Rasmussen, M. D.; Thostrup, P.; Molina, L. M.; Laegsgaard, E.; Stensgaard, I.; Hammer, B.; Besenbacher, F. Surf. Sci. 2005, 598, 226245.

(18) Lauritsen, J. V.; Foster, A. S.; Olesen, G. H.; Christensen, M. C.; Kühnle, A.; Helveg, S.; Rostrup-Nielsen, J. R.; Clausen, B. S.; Reichling, M.; Besenbacher, F. Nanotechnology 2006, 17, 3436-3441.

(19) Zhang, Z.; Bondarchuk, O.; Kay, B. D.; White, J. M.; Dohnalek, Z. J. Phys. Chem. B 2006, 110, 21840-21845.

(20) Bikondoa, O.; Pang, C. L.; Ithnin, R.; Muryn, C. A.; Onishi, H.; Thornton, G. Nat. Mater. 2006, 5, 189-192.

(21) Allred, A. L. J. Inorg. Nucl. Chem. 1961, 17, 215-221.

(22) Cordero, B.; Gomez, V.; Platero-Prats, A. E.; Reves, M.; Echeverria, J.; Cremades, E.; Barragan, F.; Alvarez, S. Dalton Trans. 2008, 21, 28322838.

(23) Shannon, R. D. Acta Crystallogr., Sect. A 1976, 32, 751-767.

JP901626B 